DOI: $10.15593 / 2224-9982 / 2017.48 .02$

УДК 621.454.3.03:539.319:536.463

\author{
В.В. Яковина ${ }^{1,2}$, В.И. Малинин ${ }^{2}$ \\ ${ }^{1}$ АО «Научно-исследовательский институт полимерных материалов», Пермь, Россия \\ ${ }^{2}$ Пермский национальный исследовательский политехнический университет, Пермь, Россия
}

\author{
УЧЕТ ДЕФОРМАЦИИ ЗАРЯДА ТВЕРДОГО ТОПЛИВА \\ ПРИ РАСЧЕТЕ ВНУТРИБАЛЛИСТИЧЕСКИХ ПАРАМЕТРОВ
}

\begin{abstract}
Для обеспечения точности расчета внутрибаллистических характеристик двигателя с зарядом из высоконаполненного смесевого твердого топлива отмечена необходимость учета эффекта существенного локального увеличения скорости горения топлива в условиях напряженно-деформированного состояния. Рассматривается прочноскрепленный заряд с канально-щелевой геометрией в цилиндрическом корпусе, пребывающий в напряженно-деформированном состоянии от действия температурной нагрузки и внутрикамерного давления. Уточняется типовой эмпирический закон скорости горения, включаемый в систему уравнений внутренней баллистики ракетного двигателя на твердом топливе. Корректировка осуществляется введением добавочного слагаемого, зависящего от осредненной первой главной деформации рабочей поверхности. Предварительно определяется математическая зависимость изменения деформации поверхности заряда по мере выгорания свода топлива. Показано, что для канально-щелевой конструкции значительные растяжения топлива локализованы в нещелевой зоне с цилиндрическим каналом. При расчете внутрибаллистических характеристик скорость горения в данной области задается по уточненному закону. Представлены результаты расчета внутрикамерного давления в сравнении с экспериментальными данными с учетом и без учета влияния деформации на скорость горения топлива в исследуемом изделии. На основании полученных данных проведена оценка применимости описанного подхода к зарядам из высоконаполненных топлив, определены погрешность и характер сходимости расчета с опытом соответственно по среднеинтегральному и текущему давлению, а также по полному времени работы двигателя.

Ключевые слова: заряд, высоконаполненное смесевое ракетное твердое топливо, напряженно-деформированное состояние, скорость горения, внутренняя баллистика, ракетный двигатель.
\end{abstract}

\author{
V.V. Yakovina ${ }^{1,2}$, V.I. Malinin ${ }^{2}$ \\ 1 JSC "Research Institute of Polymeric Materials", Perm, Russian Federation \\ ${ }^{2}$ Perm National Research Polytechnic University, Perm, Russian Federation
}

\title{
CALCULATION OF INTERNAL BALLISTICS PARAMETERS WITH CONSIDERATION OF THE SOLID PROPELLANT GRAIN DEFORMATION
}

To ensure computational accuracy of internal ballistics parameters of rocket motor with high-filled composite solid propellant it is necessary to take into account effect of local increase of burning rate in deflected mode. The authors investigate case-bonded propellant grain with bore-slot geometry and cylindrical body in deflected mode under thermal load and chamber pressure. It is refined the standard empirical law of solid propellant burning rate, which is included in equations of internal ballistics of solid rocket motor. This law is adjusted by introduction of extra component, which depends on averaged first main deformation of working surface. Preliminary mathematical relationship between grain surface deformation and grain web burning-out has been determined. Obtained results have shown that significant propellant stretching is localized in non-slot zone with cylindrical bore. In calculating characteristics of internal ballistics the burning rate in this area is assigned according to the refined law. The article contains comparative data between chamber pressure and experimental values with and without influence of deformation on the propellant burning rate in investigated item. Based on obtained data the described approach was investigated to evaluate its applicability to the high-filled propellant grains. It was determined calculating error and convergence behavior between calculation and experiment according to mean and current pressure as well as overall working time of the motor.

Keywords: solid propellant grain, high-filled composite solid propellant, deflected mode, burning rate, internal ballistics, rocket motor.

Совершенствование ракетных двигателей на твердом топливе (РДТТ) в направлении повышения тяговых характеристик обусловило, во-первых, применение прочноскрепленных зарядов из смесевых твердых топлив (СТT) с высокой энергетикой, а во-вторых, максимальное объемное заполнение топливом корпуса РДТТ при сохранении возможности реализации тре- 
буемого закона тяги [1]. Особенностью высокоэнергетических СТТ является их высокая степень наполнения компонентами окислителя и энергетических добавок, что в совокупности обеспечивает высвобождение значительной тепловой энергии в ходе реакции горения. В таких топливах массовая доля полимерного горючего-связующего, выполняющего, в частности, функции механического соединения компонентов в твердой структуре и обеспечения в процессе эксплуатации требуемого уровня физико-механических характеристик, близка к предельному уровню около $10 \%[2,3]$. Физико-механические характеристики таких СТТ находятся на уровне, достаточном для обеспечения прочностной работоспособности заряда твердого топлива (3ТT) в широком температурном диапазоне эксплуатации $\pm 50{ }^{\circ} \mathrm{C}$, однако при горении обнаруживается существенное непрогнозируемое увеличение секундного газоприхода в начальный период работы РДТТ, что приводит к отличию реальных внутрибаллистических характеристик (ВБХ) от расчетных. Поскольку задача обеспечения запрограммированного притока газов в камеру сгорания РДТТ является ключевой, рассматриваются причины описанного явления и оценивается возможность практического применения известной из литературы модели учета.

Газоприход с поверхности заряда в единицу времени определяется следующим образом:

$$
G=S U \rho,
$$

где $S$ - текущая площадь поверхности горения, определяемая геометрией заряда; $U$ - скорость горения топлива; $\rho$ - плотность топлива.

При условии постоянства плотности топлива по объему изделия из формулы (1) следует, что возможными причинами нештатного увеличения секундного газоприхода могут являться отличие реальной скорости горения топлива от расчетной, а также незапланированное развитие поверхности горения вследствие нарушения структуры, приводящее к горению непараллельными слоями. В последнем случае задача математического описания процесса горения ЗТТ значительно усложняется ввиду трудности в получении объективных данных о состоянии поверхности в процессе работы. По этой причине в настоящей работе принимается допущение о пренебрежимо малом влиянии на газоприход таких изменений структуры поверхности, которые приводят к непараллельности фронта горения в поперечном сечении заряда. Влияние на газоприход возможных микродефектов поверхности топлива учитывается путем повышения локальной расчетной скорости горения.

В большинстве литературных источников по внутренней баллистике РДТТ в качестве одного из факторов влияния на скорость горения для прочноскрепленных ЗТТ отмечается напряженно-деформированное состояние (НДС) топлива [4-6]. Однако причины роста скорости горения являются слабо изученными и подробно не рассматриваются. Физико-математические модели отсутствуют [7]. В работе [8] отмечается возможная причина увеличения скорости горения топлива в напряженном состоянии - появление сетки микротрещин, т.е. увеличение эффективной поверхности горения топлива. В настоящей статье акцентируется острота проблемы применительно к обладающим повышенной энергетикой высоконаполненным СТТ, для которых в отличие от менее наполненных (традиционных) топлив влияние НДС на горение существенно. Лабораторные эксперименты на предварительно растянутых образцах-лопаточках из высоконаполненного СТТ фиксируют существенное по сравнению с традиционными топливами увеличение текущего давления в камере опытной установки. Причины роста скорости горения деформированного топлива обусловлены особенностями строения и поведения его гетерогенной полимерно-кристаллической структуры в процессе нагружения $[9,10]$. При предельно малом или близком к таковому содержании полимерного связующего (10-15\%) образуемая им механическая связь входящих в состав СТТ порошкообразных наполнителей (кристаллических солей окислителя типа перхлората аммония, а также высокоэнергетических добавок, некоторые из которых характеризуются крайне низкой адгезией частиц к полимеру) недостаточна для удержания частиц в условиях растягивающих нагрузок. Появляются кристаллы, не связанные 
в трехмерной матрице полимера. Можно сказать, что с некоторой величины деформации растяжения в топливе происходит процесс порообразования [11].

Из вышесказанного следует, что проблема учета описанного эффекта возникает во всех случаях, когда ЗТТ пребывает при эксплуатации в состоянии, характеризуемом определенным уровнем НДС, причем минимальный уровень последнего, при котором начинает проявляться эффект для высоконаполненных СТТ, может быть крайне низким. По этой причине особенно актуальна поставленная задача учета для конструкций зарядов с высокой степенью заполнения топливом камеры сгорания РДТТ, где уровень НДС от температурной нагрузки в отрицательном диапазоне значителен. Для учета описанного трудно прогнозируемого явления разработчики только на стадии опытной отработки реальных образцов зарядов корректируют требования конструкторской документации к скорости горения топлива и вводят поправочные эмпирические коэффициенты, уменьшая тем самым погрешность расчета ВБХ. Универсального подхода к решению обозначенной проблемы на стадии эскизного проектирования не существует.

Цель настоящей работы - применение известной из литературы математической модели с линейной зависимостью от НДС к одному из типовых по конструкции ЗТТ из высоконаполненного СТТ и оценка ее практической пригодности. В качестве основного критерия была выбрана сходимость ключевых расчетных внутрибаллистических параметров РДТТ (давления в камере и времени работы) с опытными на крайних температурных режимах. Сделаны выводы относительно возможности применения данного подхода при решении практических задач.

В прочноскрепленном ЗТТ наличие НДС преимущественно обусловлено влиянием двух факторов - тепловой нагрузки и действия внутрикамерного давления $[2,11]$. При температуре, отличной от равновесной, топливный блок всегда деформирован ввиду значительного различия коэффициентов температурного расширения топлива и силовой оболочки корпуса. Появление внутрикамерного давления при запуске и последующей работе РДТТ вызывает дополнительную деформацию ЗТТ, которая суммируется с тепловой. В конструкции заряда с высокой степенью объемного заполнения камеры сгорания 80 \% и более уровень деформаций от действия перечисленных нагрузок может составлять $15 \%$ и более, что приводит к существенному повышению скорости горения высоконаполненного СТТ. Неучет этой особенности значительно увеличивает погрешность расчета ВБХ. Например, по результатам опытной отработки фиксируется превышение максимального давления в камере РДТТ по сравнению с расчетным на 15-20 \%, причем преимущественно в начальный период работы. Последнее может быть связано с тем, что степень деформированности горящей поверхности максимальна в начале работы и по мере выгорания заряда снижается, переставая оказывать влияние на скорость горения. Дополнительное искажение вида опытной зависимости давления от времени происходит в связи с неоднородностью НДС в объеме заряда. С точки зрения характеристик РДТТ непрогнозируемое увеличение скорости сказывается на требуемом

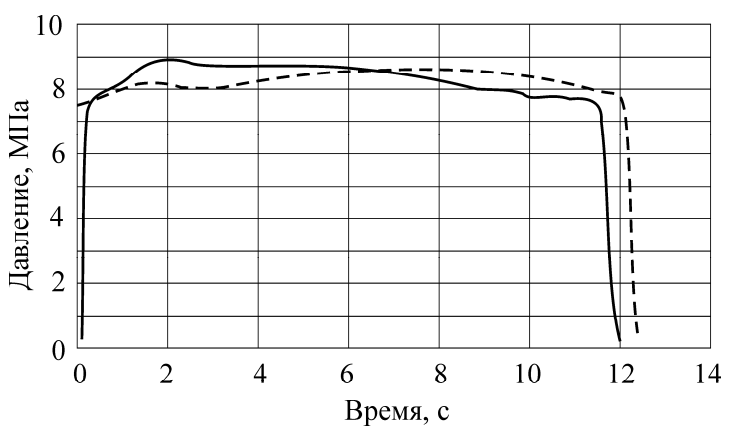

Рис. 1. Характерный вид нерасчетности при оценке внутрикамерного давления: - - опыт; - - - - расчет законе изменения тяги по времени, искажая его вид. Сокращается полное время работы двигателя.

По рис. 1 можно наблюдать характер нерасчетности. Представлены сравнительные графики расчетного и опытного давления в камере РДТТ с прочноскрепленным зарядом из высоконаполненного СТТ, обеспечивающим, по задаче проектировщика, близкий к нейтральному вид кривой давления. В условиях применения такого ЗТТ рассчитанный максимальный в начальный момент времени уровень деформации горящей поверхности 
не велик и составляет 7-10\%. Тем не менее в опыте наблюдается повышенный газоприход в начальный период работы.

Рассмотрим прочноскрепленный однорежимный ЗТТ средних габаритов с типовой канально-щелевой конструкцией и высокой степенью объемного заполнения камеры $85 \%$ (рис. 2). Стрелками обозначены направления компонент деформации в точке на горящей поверхности топлива (цилиндрическая система координат). Силовая оболочка корпуса выполнена из металла и имеет цилиндрическую форму.
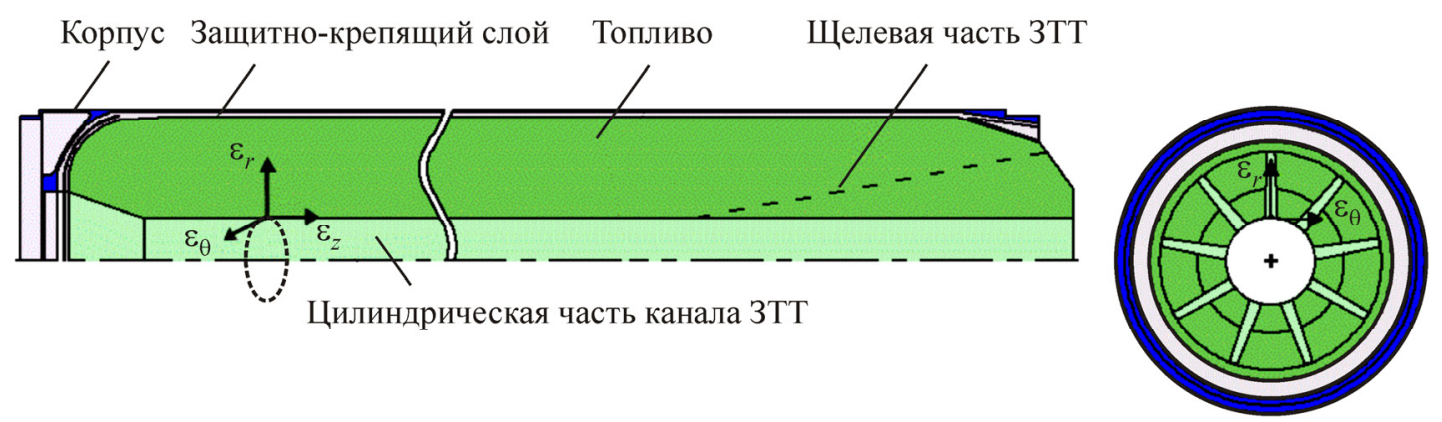

Рис. 2. Эскиз рассчитываемой конструкции ЗТТ

Зависимость относительной площади поверхности горения от толщины свода раздельно по выделенным зонам ЗТТ и суммарно по всему заряду представлена на рис. 3. Она имеет близкий к нейтральному вид, что распространено в практике проектирования. В теории тяга РДТТ с таким зарядом имеет близкое к постоянному значение с минимальным разбросом в течение всего времени работы. По рис. 3 можно оценить вклад цилиндрической и щелевой частей в величину площади поверхности (соотношение примерно 40/60 в начальный период).

Вычисление деформации ЗТТ проводилось для крайних режимов эксплуатации при совместном действии температуры -50 и $+50{ }^{\circ} \mathrm{C}$ и внутрикамерного давления 10 и 12 МПа соответственно. Характерное значение равновесной температуры рассчитываемой конструкции составляет около $45^{\circ} \mathrm{C}$, поэтому максимальный уровень НДС от тепло-

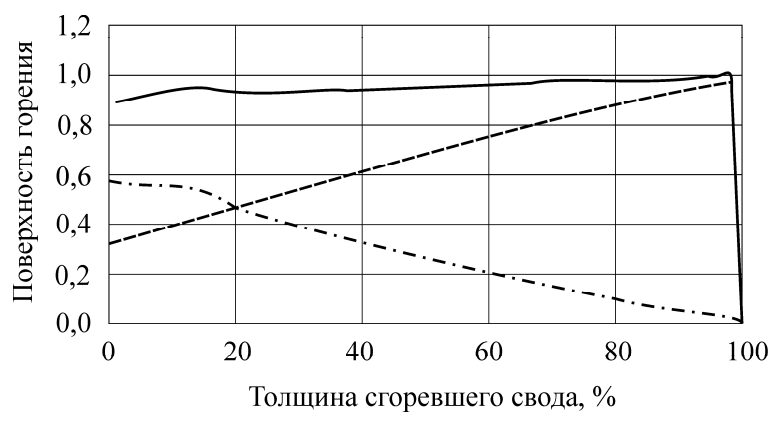

Рис. 3. Расчетная зависимость относительной поверхности горения по своду: - - суммарная; - - - - цилиндрическая часть; - - - щелевая часть вой нагрузки достигается при температуре $-50{ }^{\circ} \mathrm{C}$, когда перепад составляет $95^{\circ} \mathrm{C}$. При температуре $+50{ }^{\circ} \mathrm{C}$ напряжения в заряде от действия тепловой нагрузки отсутствуют, и поэтому общий уровень НДС определяется величиной действующего внутрикамерного давления. На рис. 4 представлены графики компонент относительных деформаций внутренней поверхности топлива по длине ЗТТ (в цилиндрической системе координат), рассчитанных в программном пакете конечно-элементного анализа (статическая осесимметричная задача). Как видно из графиков, наиболее деформированной зоной для рассматриваемых видов нагрузок является нещелевая зона с цилиндрическим каналом. Значения приведены в начальный момент работы РДТТ раздельно по виду нагрузки. Отрицательным значениям соответствует состояние сжатия топлива, положительным - растяжения.

По результатам расчета за критерий НДС горящей поверхности принята тангенциальная компонента деформации $\varepsilon_{\theta}$ (см. рис. 2), представляющая собой первую главную деформацию и характеризующая растяжение топлива [4]. Определено изменение ее суммарной, по обоим видам нагрузок, величины в процессе выгорания свода ЗТТ (реальные условия работы РДТТ). За- 
висимость имеет вид, достаточно точно описываемый экспоненциальной функцией с отрицательным показателем. На рис. 5 представлено изменение по своду осредненной по длине цилиндрической части ЗТТ компоненты $\varepsilon_{\theta}$.

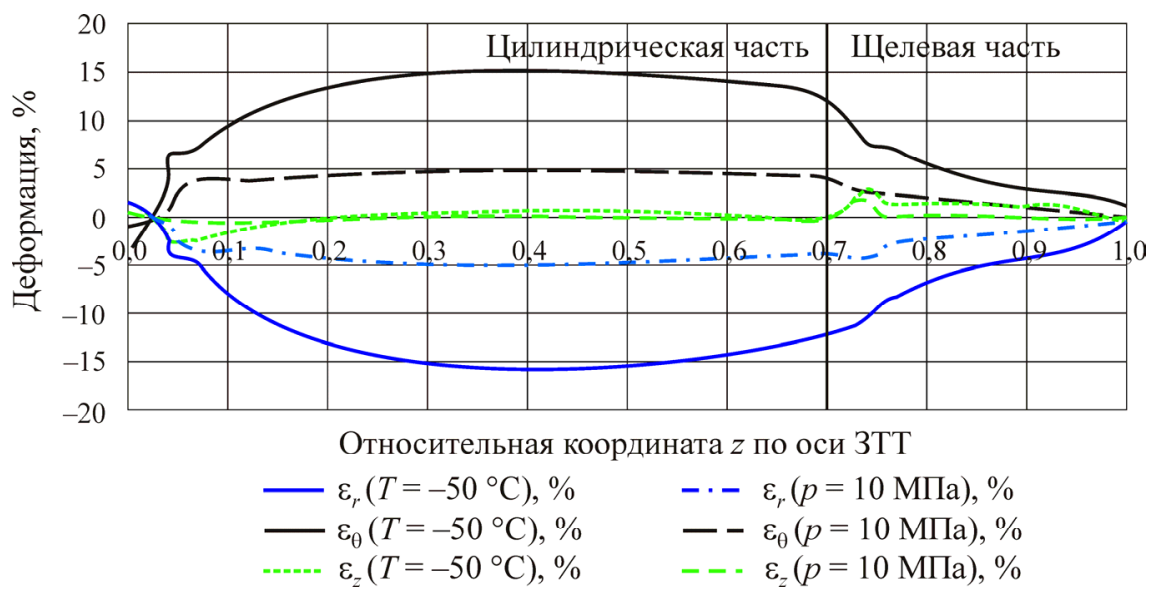

Рис. 4. Компоненты деформации поверхности топлива от температуры и давления

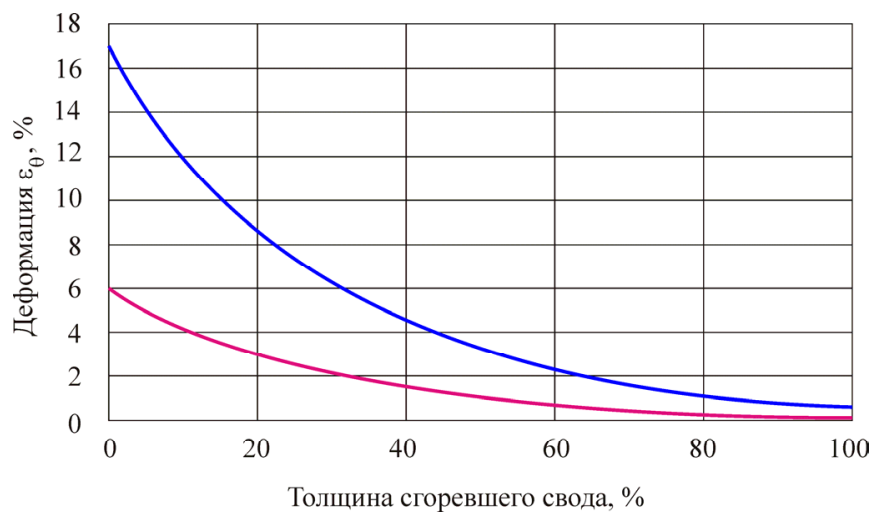

Рис. 5. Средняя деформация растяжения поверхности топлива в цилиндрической части: $--p=10 \mathrm{MПа} ; T=-50{ }^{\circ} \mathrm{C} ;--p=12 \mathrm{MПа} ; T=50^{\circ} \mathrm{C}$

Как было отмечено ранее, моделирование влияния деформации на секундный газоприход рассматриваемого заряда проводится в первом приближении. Применяемый феноменологический подход не учитывает особенностей структуры высоконаполненного СТТ и процесса горения. Проводится уточнение типового эмпирического закона скорости горения твердого топлива, включаемого в систему уравнений внутренней баллистики РДТТ [8]. Типовой закон горения твердого топлива в некотором интервале давлений в стационарных условиях описывается следующей эмпирической зависимостью [11, 12]:

$$
U(p)=U_{1} p^{v},
$$

где $U_{1}, v$ - постоянные коэффициенты, отражающие физико-химические свойства топлива и определяемые на его образцах в опытной установке; $p$ - текущее давление продуктов сгорания в камере РДТТ. Показатель $v$ характеризует зависимость скорости горения от давления. $U_{1}$ зависит также от начальной температуры заряда: 


$$
U_{1}=\frac{U_{T_{\text {оп }}}\left(1+\alpha_{T}\left(T_{3}-T_{\text {оп }}\right)\right)}{p_{\text {оп }}^{v}},
$$

где $U_{T_{\text {оп }}}$ - скорость горения топлива при постоянном давлении $p_{\text {оп }}$ и температуре образца $T_{\text {оп }}$ в опытной установке; $\alpha_{T}$ - эмпирический коэффициент зависимости от температуры; $T_{3}-$ начальная температура заряда.

Для учета влияния НДС в формулу (2) вводится множитель, связанный с выбранным ранее критерием $[13,14]$ :

$$
U\left(p, \bar{\varepsilon}_{\theta}\right)=U_{1} p^{v}\left(1+A \bar{\varepsilon}_{\theta}(\Delta E)\right),
$$

где $A$ - коэффициент, зависящий от марки СТТ и определяемый по опытам на образцах в растянутом состоянии или расчетно-аналитическим путем с использованием результатов стендовой отработки натурных изделий-аналогов; $\bar{\varepsilon}_{\theta}(\Delta E)$ - первая главная деформация на поверхности цилиндрического канала заряда, осредненная по его длине и рассчитанная для текущей толщины $\Delta E$ сгоревшего свода.

Хотя по сути величина $\bar{\varepsilon}_{\theta}$ зависит также от изменяющегося давления в камере сгорания в процессе работы двигателя, учет текущих значений давления значительно усложняет расчеты, поскольку в таком случае должна решаться совместная задача расчета НДС и ВБХ. В связи с этим принимается упрощение и учитывается зависимость от среднего давления за начальный период работы РДТТ, характеризуемый, как было отмечено ранее, высоким уровнем деформации. Для рассматриваемого ЗТТ среднее значение давления в начальный период времени составляет около 10 МПа при температуре $T_{3}=-50{ }^{\circ} \mathrm{C}$ и около 12 МПа при температуре $T_{3}=+50{ }^{\circ} \mathrm{C}$. Вычисление деформаций проводилось при данных условиях. Допустимость вводимого упрощения обусловлена также характерной конструкцией РДТТ с мало изменяющимся давлением в процессе работы. Как отмечалось ранее, динамика нагружения давлением не учитывалась (статическая задача).

В итоге в базовую эмпирическую формулу (2) включена линейная зависимость от изменяющейся по своду ЗТТ осредненной деформации $\bar{\varepsilon}_{\theta}$.

Отмечается, что для решения поставленной задачи по проверке применимости описываемого в настоящей работе подхода (физико-математической модели) разработка методики расчета переходного коэффициента $A$ не требовалась и является темой отдельных исследований. Один из вариантов приближенного определения коэффициента при отсутствии возможности проведения специальных экспериментов - расчетно-аналитический по известным опытным данным изделий-аналогов из той же марки СТТ [8]. В настоящей работе для рассматриваемого ЗТТ значение коэффициента $A$ выбиралось из условия сходимости расчетного и опытного давлений в начальный период работы РДТТ при температуре $T_{3}=-50{ }^{\circ} \mathrm{C}$. Тем самым априори принималось положение о достоверности проверяемой физико-математической модели в данный период.

Формула (3) с рассчитанными коэффициентами для рассматриваемого ЗТТ из высоконаполненного СТТ при температурах $T_{3}= \pm 50{ }^{\circ} \mathrm{C}$ имеет вид

$$
\begin{aligned}
& U^{T_{3}=+50{ }^{\circ} \mathrm{C}}\left(p, \bar{\varepsilon}_{\theta}\right)=0,16\left(\frac{p}{10^{-2} g}\right)^{0,45}\left(1+0,018 \bar{\varepsilon}_{\theta}^{T_{3}=+50{ }^{\circ} \mathrm{C}}(\Delta E)\right), \\
& U^{T_{3}=-50{ }^{\circ} \mathrm{C}}\left(p, \bar{\varepsilon}_{\theta}\right)=0,14\left(\frac{p}{10^{-2} g}\right)^{0,45}\left(1+0,018 \bar{\varepsilon}_{\theta}^{T_{3}=-50{ }^{\circ} \mathrm{C}}(\Delta E)\right),
\end{aligned}
$$


где $\bar{\varepsilon}_{\theta}^{T_{3}=+50^{\circ} \mathrm{C}}(\Delta E)=6,05 e^{-0,37 \Delta E} ; \bar{\varepsilon}_{\theta}^{T_{3}=-50^{\circ} \mathrm{C}}(\Delta E)=16,92 e^{-0,37 \Delta E} ; g$ - нормальное ускорение силы тяжести.

На рис. 6 изображены графики изменения скорости горения по своду, определенной по формулам $(4 \mathrm{a}, 46)$ при фиксированных значениях давления $p$. Для сравнения приведены значения без учета деформации. Как видно из графика, увеличение скорости при отрицательной температуре значительнее, чем при положительной ввиду более высокого уровня НДС (см. рис. 5). Далее при расчете ВБХ конструкции (см. рис. 2) давление в камере $p$ является переменной величиной.

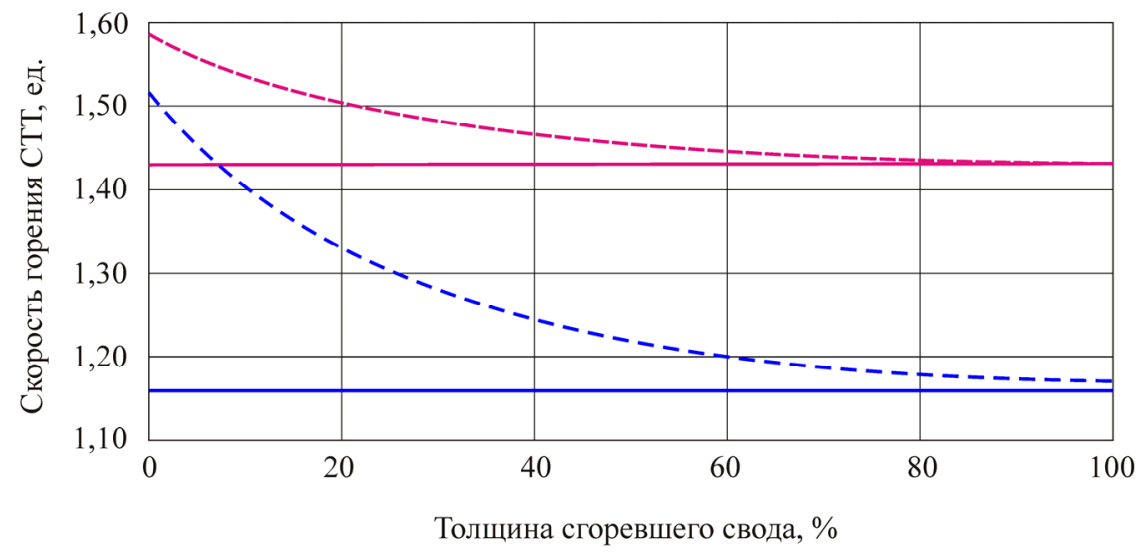

$\begin{aligned}-T & =-50^{\circ} \mathrm{C}, p_{\mathrm{cp}}=10 \text { МПа без учета деф. }--T=-50^{\circ} \mathrm{C}, p_{\mathrm{cp}}=10 \text { МПа с учетом деф. } \\ -T & =+50^{\circ} \mathrm{C}, p_{\mathrm{cp}}=12 \text { МПа без учета деф. }---T=+50^{\circ} \mathrm{C}, p_{\mathrm{cp}}=12 \text { МПа с учетом деф. }\end{aligned}$

Рис. 6. Вид зависимости расчетной скорости горения по своду

В системе автоматизированного проектирования проведен расчет внутрибаллистических параметров двигателя с рассматриваемым ЗТТ. Физико-математическая модель расчета ВБХ основана на классических уравнениях внутренней баллистики РДТТ [13, 15] с включением в систему уравнений закона скорости горения (см. (4a), (46)) применительно к цилиндрической части заряда. Скорость в щелевой части рассчитывалась по типовой зависимости (2), не учитывающей влияние НДС.

На рис. 7 представлены сравнительные графики расчетного и опытного рабочего давления в камере РДТТ в зависимости от времени работы при температурах заряда $T_{3}= \pm 50{ }^{\circ} \mathrm{C}$ с учетом и без учета деформации.
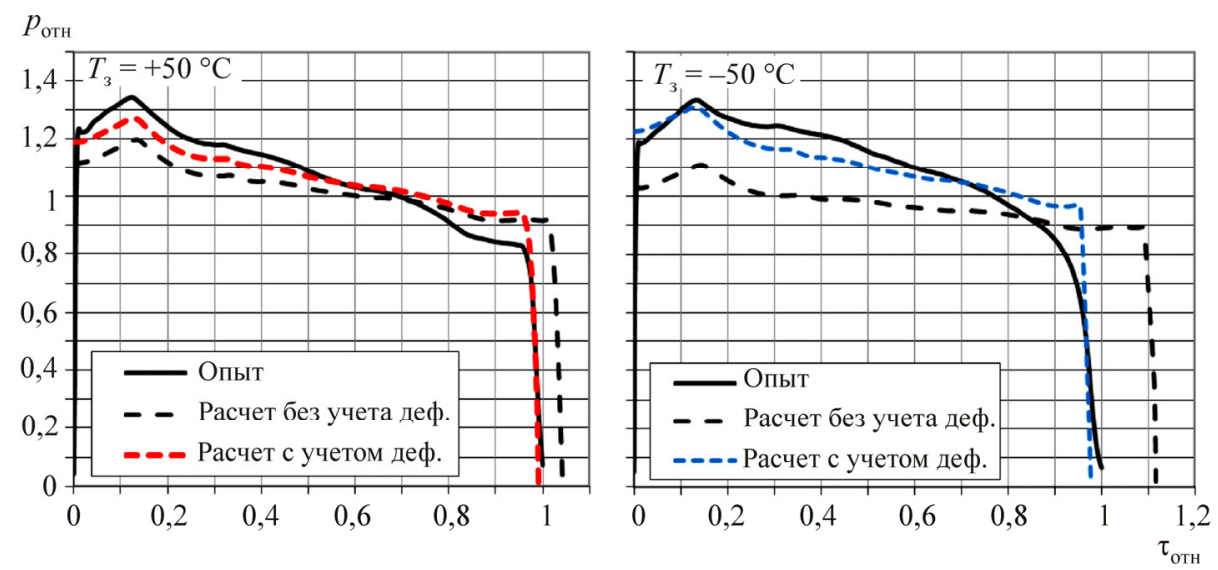

Рис. 7. Результаты расчета давления в камере РДТТ $\left(p_{\text {отн }}=p_{\text {тек }} / p_{\mathrm{cp}}^{\tau_{n}},, \tau_{\text {отн }}=\tau_{\text {тек }} / \tau_{n}\right)$ 
В таблице приведены результаты расчета относительного отклонения расчетных значений от опытных для среднего и максимального давления, полного времени работы ракетного двигателя.

Оценка относительной погрешности расчета некоторых ВБХ в сравнении с результатами натурных испытаний

\begin{tabular}{|c|c|c|c|c|}
\hline Параметр ВБХ & \multicolumn{2}{|c|}{$T_{3}=+50^{\circ} \mathrm{C}$} & \multicolumn{2}{c|}{$T_{3}=-50{ }^{\circ} \mathrm{C}$} \\
\hline & $\begin{array}{c}\text { Расчет } \\
\text { без учета } \\
\text { деформации }\end{array}$ & $\begin{array}{c}\text { Р асчет } \\
\text { с учетом } \\
\text { деформации }\end{array}$ & $\begin{array}{c}\text { Расчет } \\
\text { без учета } \\
\text { деформации }\end{array}$ & $\begin{array}{c}\text { Расчет } \\
\text { с учетом } \\
\text { деформации }\end{array}$ \\
\hline$\Delta p_{\mathrm{cp}}^{\tau_{n}}=\left(p_{\mathrm{cp}}^{\text {опыт }}-p_{\mathrm{cp}}^{\text {paчq }}\right) / p_{\mathrm{cp}}^{\text {опыт }}, \%$ & 3,6 & $-1,1$ & 10,2 & $-2,2$ \\
\hline$\Delta p_{\text {макс }}=\left(p_{\text {макс }}^{\text {опыт }}-p_{\text {макс }}^{\text {расч }}\right) / p_{\text {макс }}^{\text {опыт }} \%$ & 10,6 & 5,2 & 15 & 1,6 \\
\hline$\Delta \tau_{n}=\left(\tau_{n}^{\text {опыт }}-\tau_{n}^{\text {расч }}\right) / \tau_{n}^{\text {опыт }}, \%$ & $-4,2$ & 1,2 & $-11,4$ & 2,5 \\
\hline
\end{tabular}

По результатам проведенного исследования можно сделать следующие выводы:

1. В зарядах из высоконаполненных композиций СТТ наблюдается эффект нерасчетного увеличения секундного газоприхода при работе изделия, связываемый с изменением структуры СТТ от действия эксплуатационных нагрузок. Его неучет приводит к ощутимой погрешности определения ВБХ РДТТ.

2. Рассмотрено применение упрощенной физико-математической модели учета эффекта интенсификации процесса горения высоконаполненного СТТ в условиях НДС, позволяющей повысить точность теоретического расчета ВБХ. Применительно к РДТТ с однорежимным прочноскрепленным канально-щелевым зарядом возможное увеличение точности расчета внутрикамерного давления, достигаемое на этапе эскизного проектирования, может быть достаточным для исключения неблагоприятного исхода по прочностной работоспособности (превышению максимального давления в камере) на последующих стадиях отработки, а также для приближения тяговой характеристики к заданной.

3. Реальное применение описанной модели для увеличения точности расчета ВБХ зависит от корректного задания эмпирического коэффициента $A$ формулы (3), устанавливающего зависимость скорости горения от деформации, что сводится к разработке методики определения данного параметра.

4. Как видно из рис. 7, недостаточная сходимость по текущим значениям давления предположительно свидетельствует об упущении некоторых особенностей горения высоконаполненного СТТ в условиях НДС, которые могут быть раскрыты и учтены в ходе исследования структуры горящей поверхности и ее развития. Также на результат влияют неучтенные особенности внутрикамерного процесса конкретного исследуемого ЗТТ. Дополнительно существует погрешность от ряда допущений (по тексту статьи), принятых для упрощения проводимых расчетов.

5. В целом необходимо исследовать границы применимости рассмотренного подхода для различных по габаритам, уровню НДС и видам СТТ зарядов.

\section{Библиографический список}

1. Энергетика ракетных двигателей на твердом топливе / Ю.М. Милехин, А.Н. Ключников, Г.В. Бурский, Г.С. Лавров. - М.: Наука, 2013. - 207 с. 
2. Григорьев А.А. Введение в авиационную и ракетную технику. - Пермь: Изд-во Перм. нац. исслед. политехн. ун-та, 2014. - 176 с.

3. Косточко А.В., Казбан Б.М. Пороха, ракетные твердые топлива и их свойства. Физикохимические свойства порохов и ракетных твердых топлив. - М.: ИНФРА-М, 2013. - 400 с.

4. Алемасов В.Е., Дрегалин А.Ф., Тишин А.П. Теория ракетных двигателей. - М.: Машиностроение, 1989. - 464 c.

5. Ерохин Б.Т. Теория внутрикамерных процессов и проектирование РДТТ. - М.: Машиностроение, 1991. $-560 \mathrm{c}$.

6. Ерохин Б.Т. Теория и проектирование ракетных двигателей. - СПб.: Лань, 2015. - 608 с.

7. Внутренняя баллистика РДТТ / А.В. Алиев [и др.]; под ред. А.М. Липанова и Ю.М. Милехина; PАРАН. - М.: Машиностроение, 2007. - 504 с.

8. Яковина В.В., Малинин В.И. Учет напряженно-деформированного состояния при расчете внутрибаллистических параметров РДТТ // Аэрокосмическая техника, высокие технологии и инновации - 2015: материалы XVI Всерос. науч.-техн. конф. - Пермь: Изд-во Перм. нац. исслед. политехн. ун-та, 2015. - С. 195-198.

9. Тимнат И. Ракетные двигатели на химическом топливе: пер. с англ. - М.: Мир, 1990. - 294 с.

10. Гликман С.А. Введение в физическую химию высокополимеров. - Саратов: Изд-во Саратов. ун-та, 1969. - $380 \mathrm{c.}$

11. К вопросу выбора топлива по энергетическим и физико-механическим свойствам для зарядов РДТТ, работающих в широком температурном диапазоне / Г.Н. Амарантов, Н.М. Кочнева, П.К. Колач, А.В. Гуляев // Ракетно-космические двигатели и энергетические установки: науч.-техн. сб. / Исслед. центр им. М. В. Келдыша. - М., 2014. - Вып. 3 (160). - С. 206-214.

12. Орлов Б.В., Мазинг Г.Ю. Термодинамические и баллистические основы проектирования ракетных двигателей на твердом топливе. - М.: Машиностроение, 1979. - 392 с.

13. Соркин Р.Е. Теория внутрикамерных процессов в ракетных системах на твердом топливе: внутренняя баллистика. - М.: Наука, 1983. - 288 с.

14. Волков Е.Б., Сырицын Т.А., Мазинг Г.Ю. Статика и динамика ракетных двигательных установок. Кн. І. Статика. - М.: Машиностроение, 1978. - 240 с.

15. Шишков А.А., Панин С.Д., Румянцев В.В. Рабочие процессы в ракетных двигателях твердого топлива. - М.: Машиностроение, 1989. - 239 с.

\section{References}

1. Milekhin Yu.M., Klyuchnikov A.N., Burskiy G.V., Lavrov G.S. Energetika raketnykh dvigateley na tverdom toplive [Energy properties of solid rocket motors]. Moscow, Nauka, 2013, 207 p.

2. Grigorev A.A. Vvedenie v aviatsionnuyu i raketnuyu tekhniku [Introduction in aviation and rocket engineering]. Perm, Permskiy natsionalniy issledovatelskiy politekhnicheskiy universitet, 2014, $176 \mathrm{p}$.

3. Kostochko A.V., Kazban B.M. Porokha, raketnye tverdye topliva i ikh svoystva. Fiziko-khimicheskie svoystva porokhov i raketnykh tverdykh topliv [Powders, solid propellants and their properties. Physicochemical properties of powders and solid propellants]. Moscow, INFRA-M, 2013, $400 \mathrm{p}$.

4. Alemasov V.E., Dregalin A.F., Tishin A.P. Teoriya raketnykh dvigateley [The theory of rocket engines]. Moscow, Mashinostroenie, 1989, 464 p.

5. Erokhin B.T. Teoriya vnutrikamernykh protsessov i proektirovanie RDTT [The theory of chamber processes and designing solid rocket motors]. Moscow, Mashinostroenie, 1991, 560 p.

6. Erokhin B.T. Teoriya i proektirovanie raketnykh dvigateley [The theory and designing rocket engines]. Saint Petersburg, Lan, 2015, 608 p.

7. Aliev A.V., Lipanov A.M., Milekhin Yu.M. et al. Vnutrennyaya ballistika RDTT [Internal ballistics of solid rocket motors]. Moscow, Mashinostroenie, 2007, 504 p.

8. Yakovina V.V., Malinin V.I. Uchet napryazhenno-deformirovannogo sostoyaniya pri raschete vnutriballisticheskikh parametrov RDTT [Account of deflected mode in calculation internal ballistics of solid rocket motors]. Materialy XVI Vserossiyskoy nauchno-tekhnicheskoy konferentsii "Aerokosmicheskaya tekhnika, vysokie tekhnologii i innovatsii - 2015". Perm, Permskiy natsionalniy issledovatelskiy politekhnicheskiy universitet, 2015, pp. 195-198.

9. Timnat I. Raketnye dvigateli na khimicheskom toplive [Rocket engines with chemical fuel]. Moscow, Mir, 1990, 294 p. 
10. Glikman S.A. Vvedenie v fizicheskuyu khimiyu vysokopolimerov [Introduction in physical chemistry of superpolymers]. Saratov, Saratovskiy universitet, 1969, 380 p.

11. Amarantov G.N., Kochneva N.M., Kolach P.K., Gulyaev A.V. K voprosu vybora topliva po energeticheskim i fiziko-mekhanicheskim svoystvam dlya zaryadov RDTT, rabotayushchikh v shirokom temperaturnom diapazone [Concerning fuel selection on energy and physical-mechanical properties for grains of solid rocket motors]. Raketno-kosmicheskie dvigateli i energeticheskie ustanovki: Nauchno-tekhnicheskiy sbornik, 2014, no. 3 (160), pp. 206-214.

12. Orlov B.V., Mazing G.Yu. Termodinamicheskie i ballisticheskie osnovy proektirovaniya raketnykh dvigateley na tverdom toplive [Thermodynamic and ballistic foundations of designing solid rocket motors]. Moscow, Mashinostroenie, 1979, 392 p.

13. Sorkin R.E. Teoriya vnutrikamernykh protsessov $\mathrm{v}$ raketnykh sistemakh na tverdom toplive: vnutrennyaya ballistika [The theory of chamber processes in rocket systems with solid propellants. Internal ballistics]. Moscow, Nauka, 1983, 288 p.

14. Volkov E.B., Syritsyn T.A., Mazing G.Yu. Statika i dinamika raketnykh dvigatelnykh ustanovok. Kniga I. Statika [Statics and dynamics of rocket propulsion systems. Book I. Statics]. Moscow, Mashinostroenie, 1978,240 p.

15. Shishkov A.A., Panin S.D., Rumyantsev V.V. Rabochie protsessy v raketnykh dvigatelyakh tverdogo topliva [Operation of solid rocket motors]. Moscow, Mashinostroenie, 1989, 239 p.

\title{
Об авторах
}

Яковина Василий Васильевич (Пермь, Россия) - старший научный сотрудник АО «Научноисследовательский институт полимерных материалов», аспирант кафедры «Ракетно-космическая техника и энергетические системы» ФГБОУ ВО ПНИПУ (614113, г. Пермь, Чистопольская, д. 16, e-mail: v.yakovina@gmail.com).

Малинин Владимир Игнатьевич (Пермь, Россия) - доктор технических наук, профессор кафедры «Ракетно-космическая техника и энергетические системы» ФГБОУ ВО ПНИПУ (614990, г. Пермь, Комсомольский пр., д. 29, e-mail: malininvi@mail.ru).

\begin{abstract}
About the authors
Vasiliy V. Yakovina (Perm, Russian Federation) - Senior Researcher, JSC "Research Institute of Polymeric Materials", Postgraduate Student, Department of Rocket and Space Engineering and Power Generating Systems, Perm National Research Polytechnic University (16, Chistopolskaya st., Perm, 614113, Russian Federation, e-mail: v.yakovina@gmail.com).

Vladimir I. Malinin (Perm, Russian Federation) - Doctor of Technical Sciences, Professor, Department of Rocket and Space Engineering and Power Generating Systems, Perm National Research Polytechnic University (29, Komsomolsky av., Perm, 614990, Russian Federation, e-mail: malininvi@mail.ru).
\end{abstract}

Получено 01.11.2016 